\title{
DNA Sequencing by Tn3 Insertion in Vitro: Comparison of the Shotgun and Ordered Methods
}

\author{
Farjana Jahan Fattah ${ }^{1}$, Masayuki Morita ${ }^{1}$, Satoshi Mizutani' ${ }^{2}$, \\ Kazi Riaz Fattah ${ }^{1}$ and Yoshinobu Sugino ${ }^{1 *}$ \\ ${ }^{1}$ Laboratory of Molecular Biology, Kansai Medical University, Hirakata, Osaka 573, Japan \\ and ${ }^{2}$ Department of Public Health, Kansai Medical University, Moriguchi, Osaka 570, Japan \\ 'To whom correspondence should be addressed. Tel: +81-72-856-2121, Fax: +81-72-850-0733, \\ E-mail: sugino@makino.kmu.ac.jp
}

(Accepted for publication May 1, 2001)

Key Words: Sequencing, Tn 3, rph, pyrE, Attenuator.

\begin{abstract}
We sequenced an $E$. coli $6.6 \mathrm{~kb}$ fragment by using in vitro transposition reaction of $\operatorname{Tn} 3$. Tn 3 inserted randomly into the target plasmid and the ends of the transposon can be used as movable primer sites. Our selection system eliminates cointegrates and leaves only the simple insertions which makes it suitable for sequencing. We applied both the shotgun method and the ordered method in order to compare them. The shotgun method required more clones but produced the total sequence quickly by the use of a suitable software such as DNAMAN. Moreover, the redundancy of sequencing involved in shotgun method has the advantage of making the result more reliable. The ordered method requires information about the order and separation of transposon insertion sites in different clones in order to select the plasmids to be used for sequencing, but resulted in minimum redundancy. However, the amount of preliminary work required in the ordered method before actual sequencing far outweighs the benefit in the reduction in the number of clones necessary to be sequenced. The $E$. coli sequence we obtained turned out to be a recombinant between the standard database sequence (AE000441) and another sequence published by Jensen's group (X00781) within the rph gene.
\end{abstract}

In vitro transposition of a transposon can be used as a tool in the sequencing of a DNA that is too long to be sequenced in a single step from a single primer, when the transposon has little insertion site specificity; ends of the transposon can be used as "movable primer sites" inserted in different locations in the DNA. Several systems are now commercially available that are based on this principle.

Maekawa et al. ${ }^{1}$ has described an in vitro transposition system for $\mathrm{Tn} 3$. We modified their system to be suitable as a tool for use in sequencing a large DNA fragment. The modification consists in the choice of a selection system which allows only direct simple inser. tions of $\operatorname{Tn} 3$ to be selected, whereas Maekawa et al.'s system allows not only simple insertions but also cointegrates to survive. Cointergrates contain two copies of the transposon, which preclude their use as a sequenc. ing aid. Our system can be used either as a shotgun method, because $\operatorname{Tn} 3$ can insert at random in DNA; or alternatively a preliminary ordering step can be included using appropriate restriction enzymes.

In this paper we used this system to determine the sequence of a $6.6 \mathrm{~kb} E$. coli BamHI DNA fragment derived from strain DOO $\mathrm{crp}^{-2}$, cloned into the plasmid vector $\mathrm{pKF}^{3}$ to give the plasmid $\mathrm{pFJK1}$. We tested both the shotgun method and a sequencing procedure including a preliminary ordering step by using restriction enzymes, in order to compare their merits and demerits.

We used delta $\operatorname{Tn} 3$ transposon ${ }^{4}$ that is derived from Tn3. Most of the transposase gene is deleted in delta Tn 3 contained in plasmid pMM2004. A cell extract was prepared from $E$. coli strain $\mathrm{Kml} 196$ harboring plasmid pMM240 that overproduces $\operatorname{Tn} 3$ transposase. Cointegrates cannot survive at $42^{\circ} \mathrm{C}$ as they have the kil gene 
A)

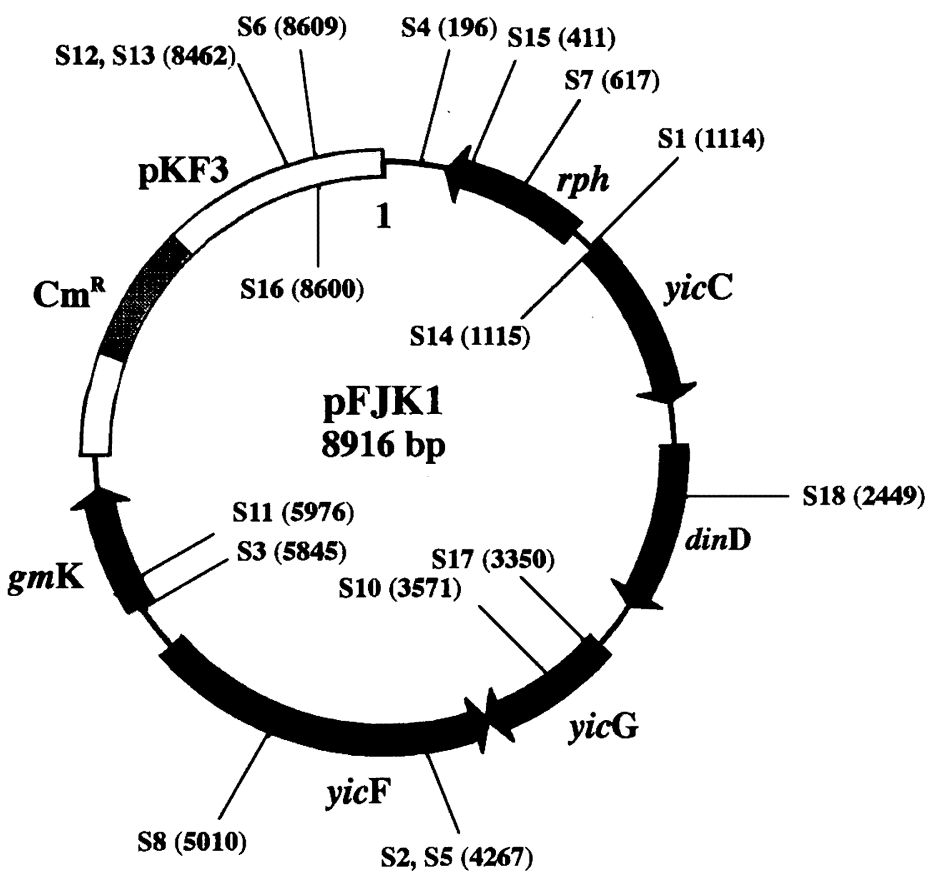

B)

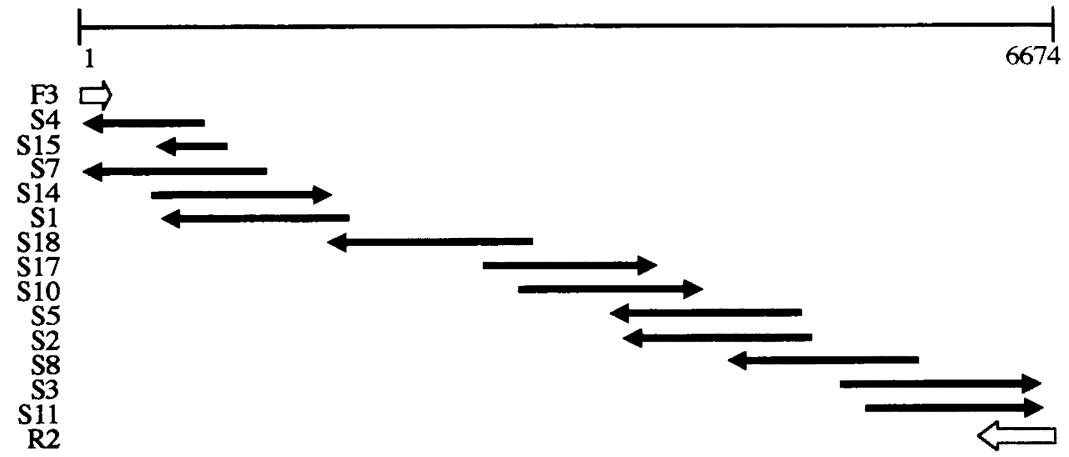

Fig. 1. The shotgun method. A) Distribution of transposon insertion sites in plasmid pFJK1. (Black Bar) E. coli 6.6 kb DNA. (Solid black arrow) Genes within the cloned E. coli DNA; rph: RNase PH, yicC: putative alpha helix protein, $\operatorname{din\mathrm {D}}$ : DNA-damage-inducible protein, yicG: orf of a hypothetical protein, yicF: putative enzyme, gmK: guanylate kinase. $\mathrm{Cm}^{\mathrm{R}}$ : chloramphenicol acetyl transferase (data obtained from $E$. coli genome database) - The numbers in the parentheses are the coordinate in our system of the site of insertion or, in the case where insertion occurred in the vector part, the vector coordinate; spikes inside the plasmid circle indicate that the transposon inserted in orientation from right to left, while those outside the circle indicate left to right orientation with respect to the coordinate of pKF3. Directions of arrows indicate directions of transcrip. tion. 1 indicates the start site of the coordinate system of the plasmid going clockwise. B) Sequence assembly graph was obtained using DNAMAN Sequence Assembly menu; parameters used were minimum overlap, 50 bases; identity between bases of the overlapping sequences, $85 \%$. The upper black line is the total sequence assembled from the different partial sequences. The names of the plasmids used are shown on the left. Primers $\mathrm{R} 2$ and F3 for plasmid pKF3 were used to sequence the ends of the $6.6 \mathrm{~kb} \mathrm{E}$. coli fragment. Sequences obtained from each delta $\mathrm{Tn} 3$ insertion with the $\mathrm{Tn} 3 \mathrm{~L}$ primer were converted into the reverse complementary sequences and after removing the 5 bp overlap, joined to the sequences obtained with the $\operatorname{Tn} 3 \mathrm{R}$ primer, so that the direction of the arrows represents the orientation of insertion of delta $\operatorname{Tn} 3$ in each clone. 
of pMM200. We used a restriction enzyme (TthIII) to eliminate the donor plasmid from the reaction mixture after transposition. This is to avoid the lethal effect of simultaneous transformation by an unreacted donor plasmid.

DNA sequencing was done with two primers corresponding to the ends of the transposon sequence and reading the sequence outward from the transposon. Joining the two sequences from the two primers for each clone gave about 1000-1600 bp sequences. Sequencing reactions were performed with BigDye terminator cycle sequencing kit (PE Applied Biosystems) and analyzed by ABI 377 automated DNA sequencer (PE Applied Biosystems). The transposon specific primers were $\mathrm{Tn} 3$ IRR (5'-GACCAAAATCCCTTAACG-3') and Tn3 IRL (5'-AGAAAACGTTGCTTAACG-3'). These primers are located near the right or left end within delta $\mathrm{Tn} 3$. For sequencing the junctions of the cloned $6.6 \mathrm{~kb} \mathrm{E}$. coli DNA with the vector pKF3, primers R2 (5'-GGACGTTTTACACCGTATTTC-3') and F3 (5'-TGCAGGAACACTCTGTTATC-3') were used. The sequence assembly and data analyses were done by DNAMAN software for Macintosh (Lynnon Biosoft).

The transposition reaction mixture $(40 \mu 1)$ contained $1.2 \mu \mathrm{g}(30 \mu \mathrm{g} / \mathrm{ml})$ donor plasmid pMM200, $0.7 \mu \mathrm{g}$ target DNA ( $\mathrm{pFJK} 1)$, the cell extract ${ }^{1}$ containing $\mathrm{Tn} 3$ transposase (protein conc. $80 \mathrm{mg} / \mathrm{ml}$ ) , $25 \mathrm{mM}$ HEPES-NaOH buffer ( $\mathrm{pH} 7.6), 60 \mathrm{mM} \mathrm{KCl}, 12 \mathrm{mM} \mathrm{Mg}$-acetate, $5 \mathrm{mM}$ dithiotheritol, $100 \mu \mathrm{g} / \mathrm{ml}$ tRNA, $50 \mu \mathrm{g} / \mathrm{ml} \mathrm{BSA}$ (bovine serum albumin), $2 \mathrm{mM} \mathrm{ATP}, 100 \mu \mathrm{M}$ each of the 4 dNTPs each, $40 \mu \mathrm{M}$ NAD, $20 \mathrm{mM}$ phosphocreatine, $3 \%$ PVA, $100 \mu \mathrm{g} / \mathrm{ml}$ creatine phosphokinase (CPK). The reaction was carried out at $30^{\circ} \mathrm{C}$ for $2.5 \mathrm{hrs}$ and stopped by adding $160 \mu \mathrm{l}$ of $50 \mathrm{mM}$ Tris- $\mathrm{HCl}$ buffer ( $\mathrm{pH} 8.0$ ) containing $10 \mathrm{mM}$ EDTA. Transposition reaction products were recovered by using QIAGEN-Mini, dissolved in $20 \mu \mathrm{l}$ water and digested by TthIII restriction enzyme to eliminate the transposon donor pMM200, followed by phenol treatment and ethanol precipitation with $4 \mu \mathrm{g}$ tRNA as carrier. After drying, the DNA was dissolved in $10 \mu \mathrm{l}$ water. $4 \mu \mathrm{l}$ of this DNA was transformed into $80 \mu \mathrm{l}$ of competent $E$. coli DOO $\mathrm{crp}^{-}$cells $^{2}$ according to Dower et al. ${ }^{3}$. The culture was shaken for $1 \mathrm{hr}$ at $30^{\circ} \mathrm{C}$ and was plated on LB plates containing chloramphenicol $(6 \mu \mathrm{g} / \mathrm{ml})$ and ampicillin $(50 \mu \mathrm{g} / \mathrm{ml})$ followed by over- night incubation at $42^{\circ} \mathrm{C}$. Thirty-three $\mathrm{Ap}^{\mathrm{R}}$ and $\mathrm{Cm}^{\mathrm{R}}$ bacterial clones containing a plasmid, all with single transposon insertion were obtained. From these 33 plasmids we chose 17 plasmids at random and performed sequencing from the transposon primers in order to test the shotgun method. The site of insertion of transposon in each clone within the total sequence was deduced which was distributed more or less evenly (Fig. 1A). The sequence information from 17 clones was more than sufficient to assemble the total sequence of the $\mathrm{E}$. coli DNA fragment (Fig. 1B).

To test the ordered sequencing method, restriction analyses of the 16 plasmids which were not used for the shotgun method were performed with three restriction enzymes BamHI, SacI and PstI to identify the approximate transposon insertion sites of each clone prior to sequencing. We knew from the known sequence data of pKF3 and delta $\mathrm{Tn} 3$ and preliminary digestion of pFJK1 that a plasmid obtained by delta $\operatorname{Tn} 3$ insertion should give three, two and three fragments, for BamHI, SacI, and PstI respectively. The lengths of restriction fragments obtained for each of these enzymes are dependent on the site of delta $\mathrm{Tn} 3$ insertion; we could calculate the approximate site of insertion of delta $\operatorname{Tn} 3$ for each clone using the restriction analyses data; actually we calculated all the 4 hypothetical outcomes of the possible positions, starting with the data of one enzyme, BamHI for example, and compared the experimentally observed data for the other two enzymes with the calculated results, to choose the right position because delta Tn3 could insert in one of two orientations and the relative orientation of the restriction fragments has two alternative possibilities. To determine the position, at least 3 restriction enzymes, viz., BamHI, SacI and PstI, had to be employed. The result also showed that in all the clones there were only single delta $\mathrm{Tn} 3$ insertions. Plasmids from two of the 16 clones contained the transposon in the vector part outside the cloned $6.6 \mathrm{~kb} E$. coli DNA, but in the other 14 the transposon was located within the $E$. coli DNA. We used the insertion sites data to select the clones distributed suitably to give the total sequence. For the sequencing we chose only nine clones, and assembled the total sequence of the cloned E. coli DNA by using the Sequence Assembly function of the DNA. MAN software (Fig. 2B). Thus ordering before sequen. 
A)

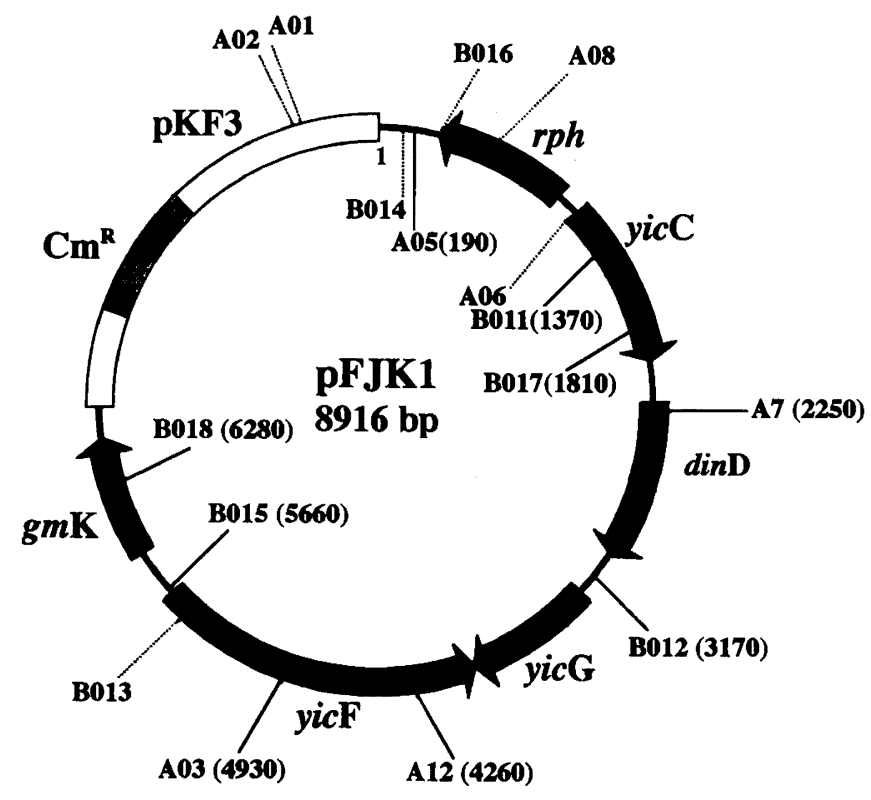

B)

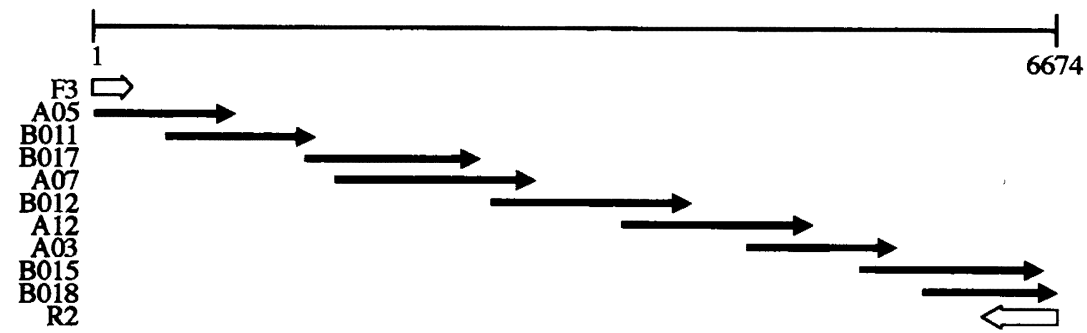

Fig. 2. The ordered method. A) Distribution of transposon insertion sites in plasmid pFJK1 used for the ordered method; those shown with dotted lines indicate clones not used for sequencing. Other explanations are similar to Fig. 1A. B) Sequence assembly graph for the ordered method. Sequences obtained from each delta $\operatorname{Tn} 3$ in. sertion with one $\mathrm{Tn} 3$ primer were converted into the reverse complementary sequences and after removing the 5 bp overlap, joined to the sequences obtained with the other primer, so that the direction of the arrows represents the direction of increase of coordinate from left to right in each clone. Other explanations are similar to Fig. 1B.

cing, though helpful to minimize redundancy of sequencing reaction, is very time-consuming and laborious. The location of the sites of insertion of delta $\mathrm{Tn} 3$ in plasmid pFJK1 in the 9 clones used in the actual sequencing in the ordered method as well as those not used (dotted lines) are shown in Fig. 2.

Thus we obtained the $6.6 \mathrm{~kb}$ sequence from both the shotgun and ordered methods. We have found that the coordinates of the ends of the $6.6 \mathrm{~kb}$ sequence are 810306 and 816979 of the $E$. coli chromosomal DNA in the coordinate system of F.R. Blattner in Genbank (Accession No. AE 000441 \& AE 000442). Our sequences obtained by both of the methods were completely identical. This sequence is identical to the database sequence except at one position; we got an extra $G$ between the position 810642 and 810647 which changed a 


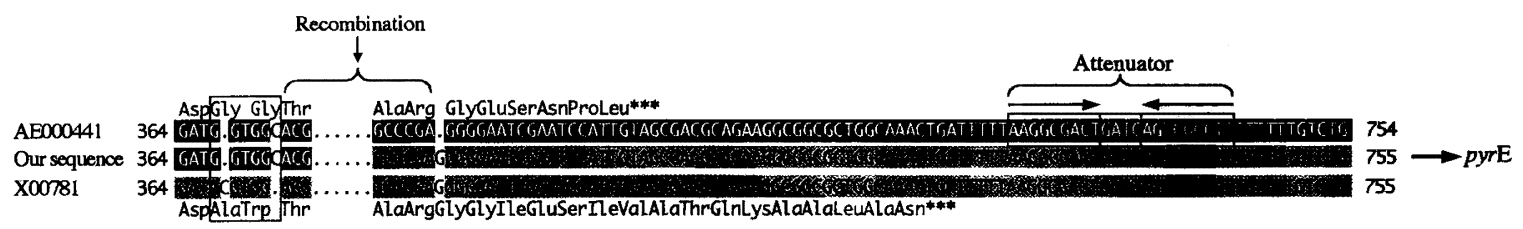

Fig. 3. Comparison of our sequence with $A E 000441^{8}$ (shown in white in black) and X00781 ${ }^{7}$ (shown in black in gray) sequences. The DNA sequence data are taken from Genbank/ EMBL/ DDBJ database (AE000441 or $\mathrm{X} 00781$ ). We count base position from the first base of the $r p h$ coding sequence. In the rph gene coding sequence we found one $C$ insertion and one $C$ deletion in comparison with $X 00781$ which change the amino acids Gly Gly to Ala Trp as shown within the box. At the end of the rph coding sequence we found an extra $G$ in comparison with AE000441, resulting in the addition of 10 amino acids, just as in X00781. Stop codons are shown with stars. The invertedly identical 9 bp sequences bracketing the 4 bp loop shown in the boxes constitute the attenuator for pyrE. Our sequence is therefore the result of a recombination event somewhere between these regions of two sequences corresponding to AE000441 and X00781.

run of $4 \mathrm{G}$ 's into a run of 5 G's.

As the two sequences obtained from the two methods showed the same difference from the database sequence it seems probable that the piece of DNA we sequenced actually had this difference from the database sequence. This would result in a frameshift of the $r p h$ gene coding sequence, in which this base is located, resulting in an $r p h$ protein 10 amino acids longer than that produced by the database sequence. As a matter of fact, Jensen? have reported that $E$. coli $\mathrm{K} 12$ strains $\mathrm{W} 3110$ and MG1655, which are used to construct the database sequence, both have a single GC pair deletion near the end of the $r p h$ gene resulting in premature termination of the RPH protein and attenuation in pyrE expression, in contrast to other strains which are without this deletion (Accession number X00781 and V01578). Thus our DNA is similar to this active form of the rph gene. However, there are two consecutive amino acid substitutions at position 123 and 124 , from Ala-Trp in the strains studied by Jensen, on the one hand, to Gly-Gly in our strain, W3110 and MG1655, on the other (Fig. 3 ). A closer look at the nucleotide sequences reveals that one can be generated by deletion and insertion of one $\mathrm{C}$ each from the other, i.e., two counteracting frameshift mutations separated by only 4 base pairs. It is thus probable that they do not affect the activity of $\mathrm{RPH}$. Thus our sequence can be considered to have re. sulted from a recombination between the AE000441 sequ. ence and X00781 sequence (Fig. 3).

This new Tn 3 based DNA sequencing strategy has allowed us to sequence the $6.6 \mathrm{~kb}$ fragment simply and rapidly. One single transposition reaction was sufficient to produce sufficient number of colonies necessary to get the total sequence both for the shotgun and ordered methods. In terms of speed the shotgun method is superior though one has to sequence more clones to get the total sequence. But it is faster and less tedious than the ordered method. The ordered method is more systematic as it selects the minimum essential number of clones prior to the actual sequencing. It thus requires a smaller number of clones to be sequenced, but the preliminary process, though straightforward, is laborious and time-consuming even in the case of a DNA of the size we chose to sequence, i.e., about $6.6 \mathrm{~kb}$. If we had to sequence a longer DNA, the assembly function of the software we used would work as successfully for the shotgun method as in the present case, but the ordering process using restriction enzymes (or PCR) could increase in complexity in a forbidding way! Thus, as long as we stick to the ordered strategy, the use of trans. poson insertion is inferior to the deletion method ${ }^{6}$. which can order partial sequences naturally in a single agarose gel electrophoresis by the size of plasmids; but the availability of an efficient computer software for sequ. 
Table 1 Comparison of Shotgun and Ordered Sequencing Strategies

\begin{tabular}{lcc}
\hline & Shotgun & Ordered \\
\hline Clones at the start & 17 & 16 \\
Number of clones sequenced & 17 & 9 \\
Total number of base pairs & & \\
used for assembly & 18740 & 10260 \\
Assembled sequence (bp) & 6674 & 6674 \\
Redundancy & 2.8 & 1.5 \\
\hline
\end{tabular}

*The average number of times a position was sequenced.

ence assembly from partial sequences makes the shotgun method with transposon insertion especially advan. tageous. Table 1 summarizes a comparison of the shotgun and ordered methods. In conclusion, the availability of an inexpensive yet powerful software has made the shotgun method the method of choice for use with the transposon insertion.

\section{References}

1) Maekawa, T., Yanagihara, K., and Ohtsubo, E. 1996, A cell-free system of $\mathrm{Tn} 3$ transposition and transposition immunity, Genes Cells, 1, 1007-1016.

2 ) Umemoto, A., Morita, M., Nakazono, N., and Sugino, Y, 1996. The effect of the crp genotype on the transformation efficiency in Escherichia coli, DNA Res., 3, 93-94.

3) Hashimoto-Gotoh, T., Tsujimura, A., Kuriyama, K., and Matsuda, S. 1993, Construction and characterization of new host-vector systems for the enforcement-cloning method, Gene, 137, 211-216.

4 ) Morita, M., Tsunasawa, S. and Sugino, Y. 1987, Overproduction and purification of the $\mathrm{Tn} 3$ transposase. J. Biochem., 101, 1253-1264.

5 ) Dower, W. J., Miller, J. F. and Ragsdala, C. W. 1988,
High efficiency transformation of $E$. coli by high voltage electroporation, Nucleic acid Res., 16, 61276147.

6 ) Morita, M., Umemoto, A., Li, Z., Nakazono, N., and Sugino, Y. 1996, Nested deletions from a fixed site as an aid to nucleotide sequencing: an in vitro system using Tn 3 transposase, DNA Res, 3, 431-433.

7) Jensen, K.F. 1993, The Escherichia coli wild type W3110 and MG1655 have an rph frameshift mutation that leads to pyrimidine starvation due to low pyrE expression levels, J Bacteriol., 175, 3401-3407.

8 ) Blattner, F. R., Plunkett, G. III, Bloch, C. A., et al., 1997, The complete genome sequence of Escherichia coli K-12, Science, 277, 1453-1474. 